\title{
Alarm Strategies and Surveillance for Mechanical Ventilation
}

\author{
Brian K Walsh and Jonathan B Waugh
}

\author{
Introduction \\ Current State of Alarm Management \\ Clinical Relevance \\ Individualization and Default Alarm Thresholds \\ Surveillance \\ Challenges in Alarm Monitoring \\ Alarm Fatigue \\ Human Factors \\ Education and Competency \\ Organization \\ Alarm Safety Culture \\ Technology Advancements \\ The Future of Alarm Management \\ Summary
}

\begin{abstract}
Clinical alarms, including those for mechanical ventilation, have been one of the leading causes of health technology hazards. It has been reported that $<15 \%$ of alarms studied rose to the level of being clinically relevant or actionable. Most alarms in health care, whether by default or intention, are set to a hypothetical average patient, which is essentially a one size fits most approach. A method of tuning to individual patient characteristics is possible, similar to the treatment philosophy of precision medicine. The excessive amount of alarms in a clinical environment is thought to be the largest contributing factor to alarm-related adverse events. All these factors come to bear on human perception and response to mechanical ventilation and clinical alarms. Observations of human response to stimuli suggest that response to alarms is closely matched to the perceived reliability and value of the alarm system. This paper provides a review examining vulnerabilities in the current management of mechanical ventilation alarms and summarizes best practices identified to help prevent patient injury. This review examines the factors that affect alarm utility and provides recommendations for applying research findings to improve safety for patients, clinician efficiency, and clinician well-being. Key words: mechanical ventilation; ventilation; alarm; alarm management; ventilator alarms; ventilator alarm management; alarms strategy; alarm surveillance. [Respir Care 2020;65(6):820-831. (C) 2020 Daedalus Enterprises]
\end{abstract}

\section{Introduction}

Mechanical ventilation is a widely used therapy in most acute care hospitals, although the execution of this life-saving therapy varies across institutions. ${ }^{1-3}$ For the past $8 \mathrm{y}$, clinical alarms have been included on the list of top 10 health technology hazards by the Emergency Care
Research Institute (ECRI). General medical device alarm hazards were listed as number one from 2012 to 2015 and as the number two hazard in 2016. By 2017, only ventilator alarms remained on the list. Today, ventilator alarms remain at number four. ${ }^{4}$ According to the Boston Globe, ${ }^{5}$ $>100$ patients who depend on mechanical ventilators have died in a 5.5-y period due to medical errors 
involving alarms; countless others have been injured. In 2010, the Food and Drug Administration received $>2,500$ adverse event reports associated with ventilator use, in which about a third of the events indicated an alarm-related issue. ${ }^{6}$ It is well known that there are inconsistencies in the practice of mechanical ventilation alarm management. Mechanical ventilation alarms and alerts provide clinicians with vital information about the device's ability to deliver the input settings and the patient's physiologic response to those settings, yet these alarms in and of themselves' have been suggested as hazardous to patient safety ${ }^{4}$ (Fig. 1).

In 2014, The Joint Commission introduced "Use alarms safely" as a National Patient Safety Goal. Phase 1 required hospitals to identify the most important alarm signals to manage. Questions like "What mechanical ventilation alarms are important?" and "Who determines if an alarm is unnecessary?" arose quickly. This drove many leaders within the respiratory therapy community to investigate best practices. Researchers from Yale University attempted to answer those questions through an integrative review of 12 previous studies published between 1986 and 2015. They reported that, when clinically irrelevant alarms were compared as a percentage of total annotated alarms, most studies revealed that only $5-13 \%$ of alarms were clinically relevant or were referred to as actionable. The definitions of clinical relevance were inconsistent across studies, which made clinical relevance of alarms difficult to determine. ${ }^{7}$

In 2016, phase 2 of the National Patient Safety Goal to use alarms safely asked hospitals to establish policies and procedures for the management of alarms identified in phase 1. Many hospitals created or updated their policies and procedures to remain compliant, yet little evidence exists as to whether this will improve the care of mechanically ventilated patients. In 2018, this goal remained as "Make improvements to ensure alarms on medical equipment are heard and responded to on time." 8 Through the National Patient Safety Goals, care facilities have faced an increasing need to manage quality improvement through innovative designs specifically targeted at using alarms safely. Reasons for lack of standardization could include regional variations, caregiver tolerance of alarm fatigue, and fear of litigation resulting from a stated standard. This review examines

The authors are affiliated with Liberty University, Lynchburg, Virginia.

Dr Walsh presented a version of this paper at the 58th Respiratory Care Journal Conference, held June 10-11, 2019, in St Petersburg, Florida.

The authors have disclosed no conflicts of interest.

Correspondence: Brian K Walsh PhD RRT, Liberty University School of Health Sciences, 1971 University Blvd, Center for Natural Sciences, Lynchburg, VA 24515. E-mail: bkwalsh@liberty.edu.

DOI: $10.4187 /$ respcare.07546 vulnerabilities in the current practice of mechanical ventilation alarm management and provides a summary of best practices identified to help prevent patient injury.

\section{Current State of Alarm Management}

Monitoring alarm systems for safety and quality largely consists of individualized monitoring practices, but management of mechanical ventilation has distinct differences that should be explored independently. Unlike bedside physiologic monitoring alarms, mechanical ventilation alarms can prevent a negative physiologic result if properly monitored and managed. For example, if a disconnect or minute ventilation alarm is quickly resolved, a cascade of negative physiologic events and their associated physiologic alarms can be prevented. The American Association for Respiratory Care has several evidence-based clinical practices and expert panel reference-based guidelines surrounding the practice of mechanical ventilation. ${ }^{9-14}$ The clinical practice guideline that influenced mechanical ventilation communication, monitoring, and safety practices today, entitled "Patient-Ventilator System Checks," was retired due to improvements in technology that made some of the positions obsolete and due to a lack of evidence for others. ${ }^{15}$ Another evidence-based clinical practice guideline has yet to take its place. This is largely due to the lack of evidence needed to meet today's evidence-based clinical practice guideline development standards. However, an issue paper published by the American Association for Respiratory Care and the University Health System Consortium's (now known as Vizient) Respiratory Care Network titled "Safe Initiation and Management of Mechanical Ventilation" was developed in 2017 and provides guidance on alarm management. ${ }^{16}$ Current recommendations include but are not limited to (1) establishing a time requirement to respond to alarms; (2) establishing a list of parameters that require monitoring; and (3) designating alarm priority levels (eg, level 1,2, or 3).

\section{Clinical Relevance}

Research suggests that $80-99 \%$ of alarms in general are false or nonactionable. ${ }^{17-22}$ Ventilator alarms are considered high risk and therefore are considered high-priority alarms in health care facilities. Both morbidity and mortality related to ventilator alarm management have been reported by the Food and Drug Administration in its MAUDE database. ${ }^{6,23}$ Although 90\% of adverse events related to mechanical ventilation alarms did not result in harm, the risk of one missed ventilator disconnect or malfunction could result in permanent harm or death. This leads us to the issue of clinical relevance. Clinical relevance can be an ambiguous and subjective term, with most institutions defining it through alarm policy. Interventions should focus on reducing 

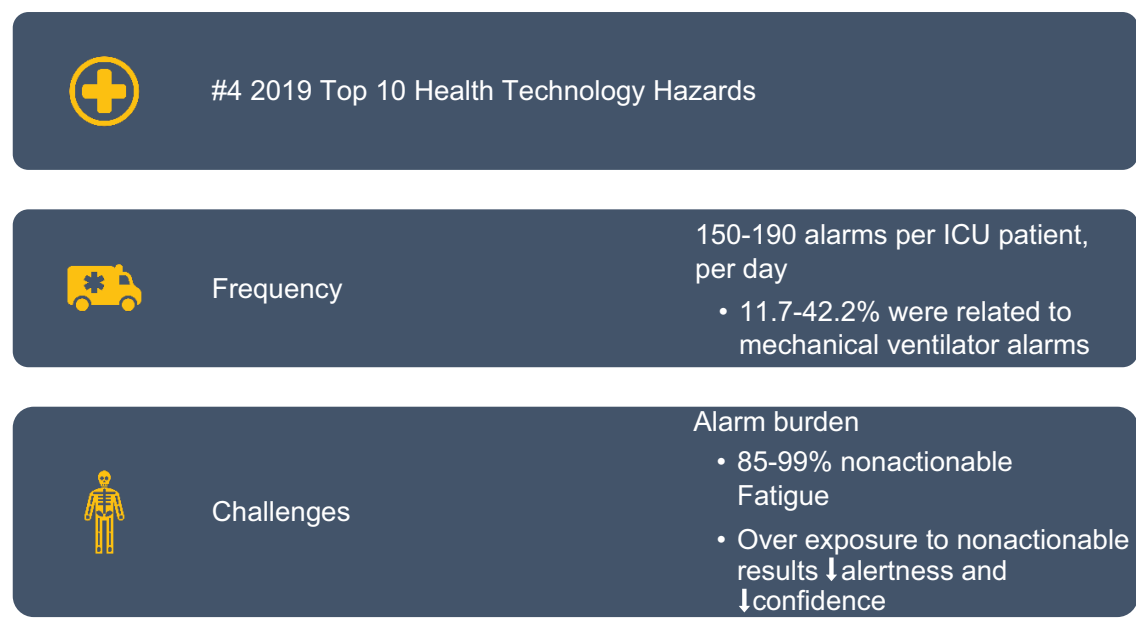

Fig. 1. Mechanical ventilator alarms have been identified as the fourth leading health technology hazard.

clinically irrelevant alarms with careful consideration for how clinical relevance is defined and measured. Most mechanical ventilation practices include setting alarms such as high pressure, disconnect, and minute ventilation. The practice of setting alarms varies widely from extremely liberal (ie, practically off) to very conservative (ie, triggering many nonactionable alarms). Both have the ability to create unsafe environments due to delayed alarming and alarm fatigue.

\section{Individualization and Default Alarm Thresholds}

Today's mechanical ventilation alarm threshold practices include individualization of most alarms to the patient's condition. Default alarms are preprogramed based on institutional policy or guidance during installation and have a variety of default settings according to patient category (eg, neonatal, pediatrics, adult) and associated modes of ventilation. However, these are often leveraged during the initial set-up as a starting point and with a balance between nonactionable and actionable alarms while admitting a patient who requires mechanical ventilation. Careful consideration should be given to justify the use of default alarms to avoid inappropriate generalization. ${ }^{16}$ After admission and stabilization of the mechanically ventilated patient, alarm thresholds are typically set to alert the clinician that the clinical goals or targets of mechanical ventilation have not been met. This attempt at individualizing the alarm threshold is designed to mitigate nuisance alarms and alarm fatigue, while creating an environment that is the safest and most responsive to individual patient care needs.

\section{Surveillance}

Clinicians, mainly respiratory therapists in the United States, monitor mechanically ventilated patients by performing patient-ventilator assessments on average every 2-4 h, with some low acuity patients going as long as $12 \mathrm{~h}$ between assessments. There is no standard approach to these assessments, likely because these visual spot checks are inadequate as a consistent and reliable safeguard in the modern patient care environment. ${ }^{24}$ We know from the literature on opioid-associated respiratory depression that there is a pattern of doubt in the ability of spot checks to prevent negative outcomes: in $42 \%$ of confirmed deaths, the interval between last nursing assessment and detection of opioid-induced respiratory depression was $<2 \mathrm{~h}$, whereas in $16 \%$ of cases the last check occurred within 15 min. $^{25}$

\section{Challenges in Alarm Monitoring}

Although there are many studies highlighting issues with alarms and their association with patient safety, studies documenting solutions are more limited. This is likely because alarm-related adverse events are multifactorial and involve patient, human, organizational, and technical factors. The risk for alarm adverse events can increase due to organizational issues such as lack of alarm accountability and a lack of agreement on setting alarms, human factors such as inconsistent response, and technical complications such as varied or confusing alarm signals (Fig. 2). ${ }^{21,26-28}$

\section{Alarm Fatigue}

The biggest contributing factor to alarm-related adverse events is suggested to be the excessive number of alarms in a clinical environment, which can be as high as 942 alarms per day. ${ }^{26}$ Regarding mechanical ventilation contributing to the environment of alarm fatigue specifically, it appears that mechanical ventilator alarms contribute to approximately a third of all alarms in the ICU (Fig. 3). ${ }^{17,18,29,30}$ Smaller studies 


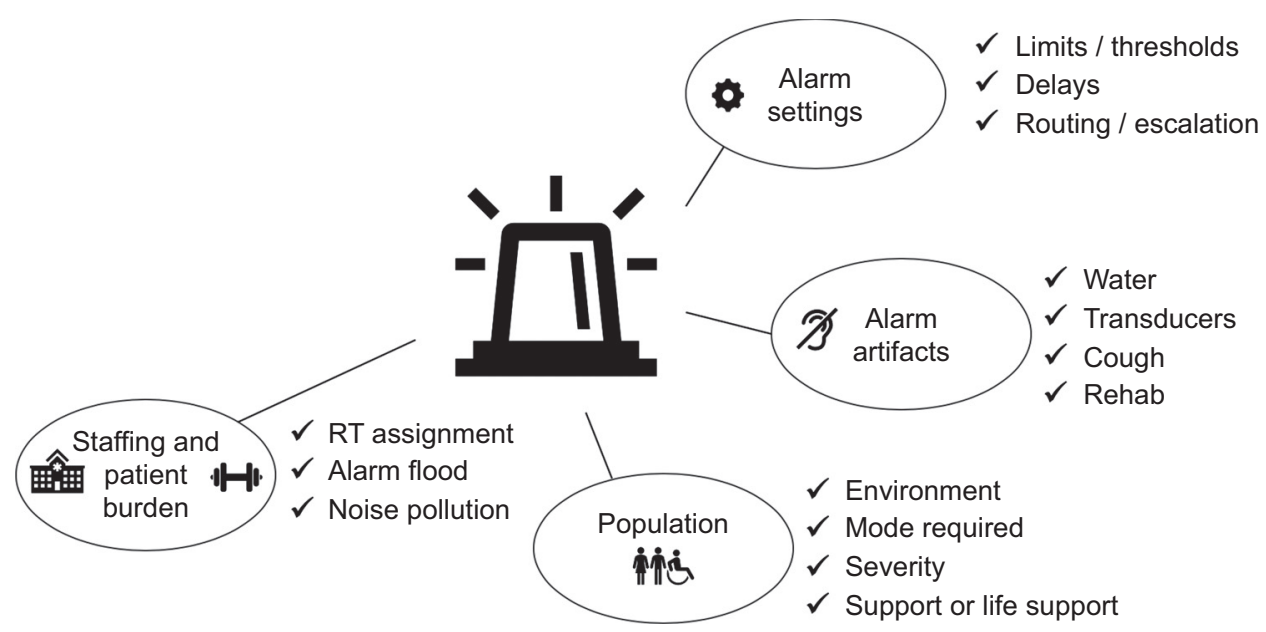

Fig. 2. Mechanical ventilator alarms are multifactorial and include 4 categories: alarm settings, alarm artifacts, population, and staffing and patient burden. $\mathrm{RT}=$ respiratory therapist.

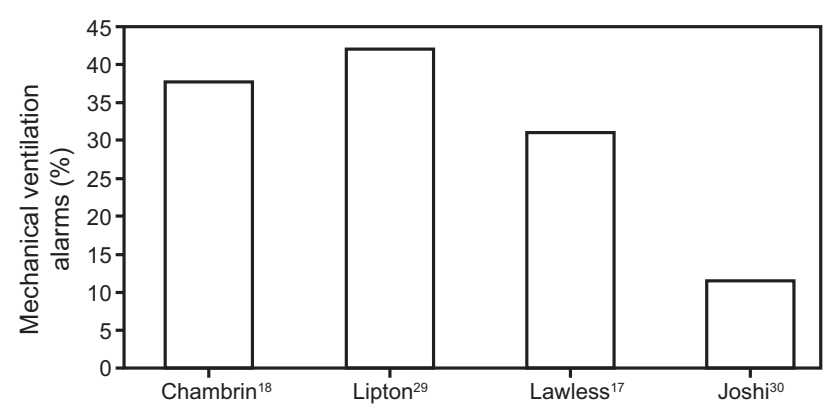

Fig. 3. Percent of alarms associated with mechanical ventilation.

have reported 7-8 alarms per ventilator hour in mechanical ventilation. ${ }^{31,32}$ More importantly, the current frequency of alarms has likely contributed to unfavorable clinician behaviors such as disabling or silencing critical alarms, setting inappropriate thresholds, or reducing alarm volumes, which have undoubtedly resulted in negative patient outcomes.

Alarm fatigue occurs when busy health care workers are exposed to frequent safety alerts or alarms and as a result become desensitized to them. This fatigue can lead to poor outcomes through longer response times or to completely missing/ignoring important or life-saving alarms. Mechanical ventilation alarms make up a third of all alarms in the ICU or approximately 9 alarms per bed per hour ${ }^{32}$ and therefore contribute to the ambient noise. Imagine trying to detect the important alarms from all the false or nonactionable alarms in a busy ICU while potentially being a few rooms away. This overwhelming task leads to risky behavior such as silencing or ignoring alarms. This completely negates the original design of alarm or alerting. This alarm fatigue not only harms health care workers, it has negative effects on patients and families as well. Alarm noise creates an unpleasant environment for patients and families. This noisy environment has been shown to delay patient recovery and increase stay. ${ }^{33,34}$ Additionally, the effects of these alarms causes the families to lose trust in the staff when they don't respond in a timely manner. The best way to treat or reduce alarm fatigue is to reduce the burden, but there is no one answer here.

\section{Human Factors}

It has been suggested humans tend to respond to alarms in the same proportion to their perceived reliability of the alarm system. ${ }^{35,36}$ Meaning if clinicians believe a particular alarm is $90 \%$ true, they respond to them $90 \%$ of the time and if it's $10 \%$ true, these alarms will be responded to about $10 \%$ of the time. Likewise, clinician response time grows with nonactionable alarms. ${ }^{22}$ In a recent survey of attitudes and practices related to clinical alarms (survey included 30\% respiratory therapists) Ruppel et $\mathrm{al}^{37}$ reported a disappointing trend in the past 10 years, including worsening perceptions of nuisance alarms and more alarm-related adverse events, and many clinicians are more likely to agree nuisance alarms occur frequently and disrupt patient care than 10 years ago. Therefore, staff performance must be measured on the basis of the work environment, and options such as extending alarm delays may be worth the sacrifice in response time if the ultimate benefit to the patient is greater.

\section{Education and Competency}

Literature suggests there is a lack of or limited training for alarm end users. ${ }^{21,26}$ Nurses, respiratory therapists and physicians receive differing levels of education regarding mechanical ventilation, alarm management and may interpret alarms and their meaning differently. Yet, they all have equal access to mechanical ventilator alarms. 
Initiating and maintaining mechanical ventilation is a complex process. Alarm settings are not universal and are often different manufacturer to manufacturer. It is imperative each clinician who manages or assists in the management of mechanically ventilated patients be educated and remain competent within their role. The clinician primarily responsible for the mechanically ventilated patient should ensure those assisting in the management are competent to help.

Alarm management knowledge and skills must start in health care education. Staff education is the bedrock of all change management efforts, yet it is often used as a last resort. Research has demonstrated that educational interventions that increase clinicians' awareness and competency with using monitoring systems can decrease alarms. ${ }^{38-42}$ While these studies are not specific to mechanical ventilation, there is no reason to believe it wouldn't be similarly effective. There is a growing movement to only monitor those items during mechanical ventilation that must be monitored for the sake of patient safety. Clinicians need to be aware that alarms are designed by default to be high in sensitivity but low in specificity because of manufacturer liability concerns. Alarm thresholds are usually set to a generalized population instead of to patient-specific conditions. In addition, policies and clinical practice tend to follow the better-safe-than-sorry logic, ${ }^{43}$ which only contributes to the problem.

Conversely, education should be targeted to provide safe, patient-specific thresholds and maintenance of the mechanical ventilation equipment to ensure the most accurate monitoring possible. Specific education should provide the true meaning of each alarm, whether it is categorized as low, medium, or high priority, and what actions should take place when an alarm occurs. Continuing education on mechanical ventilation alarms for all staff responsible for caring for these patients should be provided.

\section{Organization}

Recommendations from the Emergency Care Research Institute (ECRI), Association for the Advancement of Medical Instrumentation (AAMI), and the Joint Commission on the Accreditation of Healthcare Organizations (JCAHO) emphasize multidisciplinary teamwork and leadership as well as staff engagement to effectively reduce nuisance alarms. However, bedside alarm first responders emphasize the need for standardized alarm configuration. ${ }^{28}$ Respiratory therapists frequently are tasked with managing patients who require mechanical ventilation in hospitals. To be effective in using alarms safely, the development of best practices and protocols should focus on navigating how to create value-added alarms rather than additional alarms. ${ }^{44}$ In the process of defining best practices for reducing unnecessary alarms, the National Coalition for Alarm Management Safety found that a small number of patients are responsible for triggering most alarms and thus targeting interventions to those identified patients would substantially reduce nuisance alarms. ${ }^{45}$ Specific to mechanical ventilation management, the leading causes for ventilator alarms and sentinel events with mechanical ventilation is the lack of adaptations of the alarm thresholds when ventilator modes were changed. ${ }^{46}$

\section{Alarm Safety Culture}

It is important to promote an alarm safety culture involving administration. Ursprung et $\mathrm{al}^{47}$ reported that promoting a blame-free culture facilitated acceptance by staff when implementing new interventions. Specifically, engaging staff in identifying alarm problems and developing a solution was suggested as crucial to solving and promoting an alarm safety culture. A policy holding staff accountable is also crucial in the prevention of noncompliance. Real-time audits have demonstrated a continued need to correct human errors and behaviors. In fact, one study reported that alarm compliance improved by $84 \%$ by conducting audits. ${ }^{48}$ In most institutions this can be easily added to currently existing core safety elements.

Those with a good mechanical ventilation alarm safety culture often have:

- Comprehensive interdisciplinary mechanical ventilation alarm management policy and procedure

- Patient-specific alarm thresholds

- Documentation of the alarm thresholds

- Procedures for pausing or silencing alarms

- Standard procedures for evaluating safe mechanical ventilation alarm management and response

- Routine alarm inventory and prioritization

- Measuring mechanical ventilation alarm frequency through technology or safety rounds

- Adjusting time standards or staffing models that consider mechanical ventilation alarm response time

- Ensuring accountability of mechanical ventilation alarms

- Education on proper mechanical ventilation maintenance to ensure accurate measurements and reduce artifacts

- Surveillance of mechanical ventilation frequency and standardized interventions when frequency exceeds predetermined standards

- Education on the frequency and types of mechanical ventilation modes and brands to all clinicians 
Table 1. Ventilator alarm offerings by manufacturer

\begin{tabular}{|c|c|c|c|c|c|c|c|c|c|}
\hline Ventilator & $\begin{array}{c}\text { Tidal } \\
\text { Volume }\end{array}$ & Frequency & $\begin{array}{l}\text { Minute } \\
\text { Volume }\end{array}$ & $\begin{array}{l}\text { Airway } \\
\text { Pressure }\end{array}$ & Apnea & Disconnect & Delays & $\mathrm{FI}_{\mathrm{O}_{2}}$ & Autoset \\
\hline $\begin{array}{l}\text { Puritian Bennett } \\
\quad 980\end{array}$ & Inspiratory & Total & $\begin{array}{l}\text { Total } \\
\text { Mandatory } \\
\text { Spontaneous }\end{array}$ & Peak & Adjustable & $\begin{array}{l}\text { Circuit } \\
\text { Power } \\
\text { Gas pressure }\end{array}$ & Escalation protocol & MPS & \\
\hline $\begin{array}{l}\text { Hamilton } \\
\text { G5 }\end{array}$ & Expiratory & Total & Total & $\begin{array}{l}\text { Peak } \\
\text { PEEP }\end{array}$ & Adjustable & $\begin{array}{l}\text { Circuit } \\
\text { Power } \\
\text { Gas pressure }\end{array}$ & & MPS or adjustable & All alarms \\
\hline $\begin{array}{l}\text { Dräger } \\
\text { C500 }\end{array}$ & Expiratory & Total & Total & $\begin{array}{l}\text { Peak } \\
\text { PEEP }\end{array}$ & Adjustable & $\begin{array}{l}\text { Circuit } \\
\text { Power } \\
\text { Gas pressure }\end{array}$ & Minute ventilation & MPS & All alarms \\
\hline Servo U & N/A & Total & Total & $\begin{array}{l}\text { Peak } \\
\text { PEEP }\end{array}$ & Adjustable & $\begin{array}{l}\text { Circuit } \\
\text { Power } \\
\text { Gas pressure }\end{array}$ & & MPS & All alarms \\
\hline Avea & Expiratory & Total & Total & $\begin{array}{l}\text { High peak } \\
\text { Low peak } \\
\text { PEEP }\end{array}$ & Adjustable & $\begin{array}{l}\text { Circuit } \\
\text { Power } \\
\text { Gas pressure }\end{array}$ & & & \\
\hline
\end{tabular}

- Routine re-evaluation of the mechanical ventilation alarm assessment and evaluation frequency

- Modifying as needed the policy and procedure on mechanical ventilation alarm management

\section{Technology Advancements}

Strategies to bring about meaningful change often include technology. Advancements in technology have shown promise in helping with mechanical ventilation alarm management when they target real problems such as alarm settings, artifact management, individualization and take into consideration staff and patient burden as outlined in Figure 2.

Alarm Thresholds. Table 1 demonstrates how ventilator manufacturers have taken different approaches to alarming. The majority of manufacturers provide the ability to program default alarm thresholds based on various patient populations. Many allow you to set alarms with an autoset feature, which works by allowing the threshold to be set automatically with the push of a button to a predefined setting, such as $\pm 30 \%$ of the current minute ventilation. This feature helps providers set alarm thresholds quickly and reduces human error.

Some ventilators provide the ability to set alarm thresholds for peak inspiratory pressure, tidal volume, frequency, and minute ventilation, while others do not provide a tidal volume alarm or other limits. Few devices allow you to alarm a single variable 3 different ways (eg, minute ventilation alarms: total, demand, and spontaneous). This can lead to standardization difficulties when you have multiple brands of ventilators that may provide too many options or too much variation. Gorges et $\mathrm{al}^{46}$ reported that the Servo 300 ventilator, which only alarmed minute ventilation, had significantly fewer alarms per hour than comparator ventilators (Puritan Bennnett 840, Avea), which alarmed frequency, tidal volume, and minute ventilation. In addition, clinicians often feel the need to set all available alarms when the manufacturer makes them available. However, alarming a single variable such as pressure in multiple ways (eg, high pressure, low pressure, PEEP) has proven beneficial when determining vital events such as ventilator disconnects and partial or full occlusion of the ventilator circuit.

Smart Alarms. There is an evolving concept of creating intelligent alarms. Some of the basic ideas are alarms and options that default to clinician input such as patient category, weight, and mode of ventilation. The escalation of alarm priority (visual and tone) or auditory volume over time if not resolved or silenced within a specific timeframe makes these alerts hard to ignore and more audible if distance or isolation is an issue. Additionally, coupling of alarms such as low tidal volume with low pressure alarms can accurately signify the highest priority disconnect alarm than a stand-alone low-pressure alarm. These alarms have been coined "smart alarms" because of their inputs or algorithmic output.

Another type of smart alarm can be silenced ahead of time with clinician input such as with suctioning. Several mechanical ventilator manufacturers allow the selection of a suctioning feature, which silences certain alarms for a period (usually $2 \mathrm{~min}$ ) while preoxygenating the patient and then returns to normal operation when the patient is reconnected to the ventilator. 

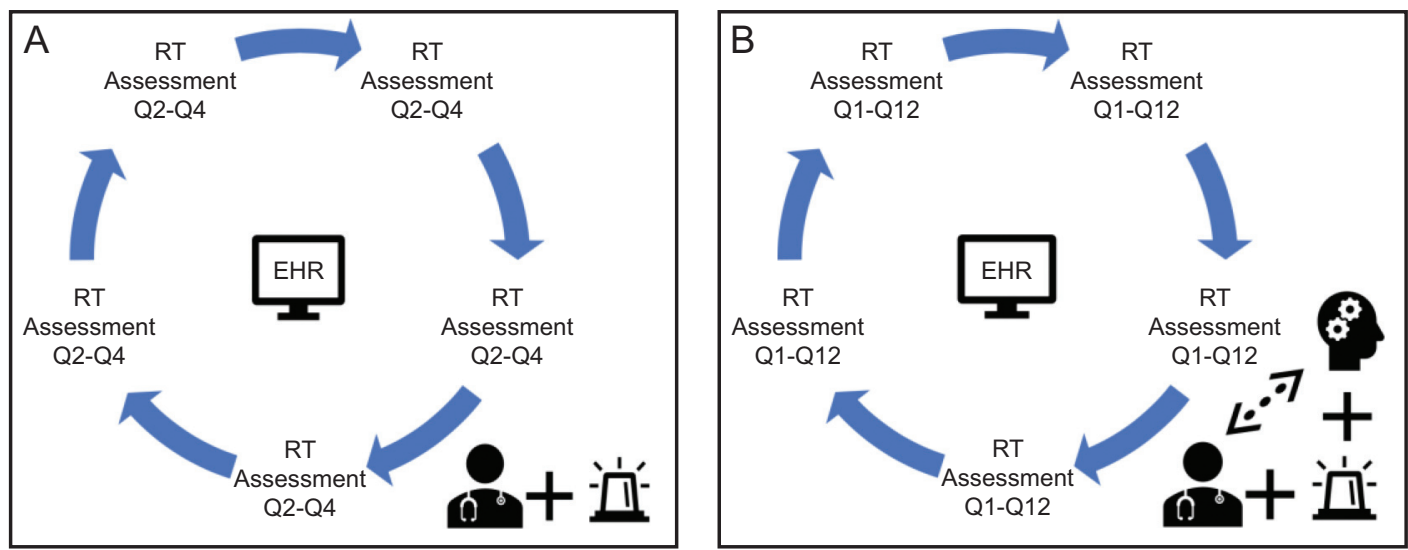

Fig. 4. Surveillance of mechanical ventilation practices. A: Current practice of intermittent surveillance (ie, ventilator patients assessed every 2-6 h). B: Potential future of continuous surveillance (ie, no interval of ventilator patient assessment, occurs as needed) leveraging remote monitoring technology. EHR = electronic health record; RT = respiratory therapist.

Adding an alarm delay has been considered to reduce nonactionable alarms and potentially to reduce alarm fatigue, although this delay might improve alarm accuracy at the expense of lengthening alarm response time. An alarm delay of $6 \mathrm{~s}$ with pulse oximetry can reduce alarms by half. ${ }^{49}$ Likewise, introducing a 19-s alarm delay of all alarms, including mechanical ventilation, could reduce alarms by $67 \%$ in the medical ICU. ${ }^{46}$ If an automatic system capable of detecting events such as turning, oral care, suctioning and bathing was integrated, alarms could be reduced by $71 \%{ }^{46}$

\section{The Future of Alarm Management}

The future of alarm management will challenge traditional safety norms. One of the best methods to eliminate alarm management issues is to prevent alarms. To do this, we will need to be proactive in determining which alarms add value and to leverage technology along with behavioral science to create a culture of safety. One of the first challenges is the practice of patient-ventilator assessment and safety rounds. Traditionally respiratory therapists' patientventilator assessments occur every $2-6 \mathrm{~h}$ while a patient is on mechanical ventilation, or as needed, and mechanical ventilation safety rounds (ie, rounds that specially look at alarm function and safety equipment) occur once per shift. While this is current practice, respiratory therapists spend countless hours documenting numbers that often do not add value or demands they spend time with patients who don't require so much time just because it is policy. These intermittent assessments and safety rounds do not address true safety issues. Patients who require mechanical ventilation to survive require continuous surveillance.

Continuous Surveillance. In 2016, the cause of death of an estimated 251,000 patients was reported to be due to medical error. ${ }^{50}$ Yet health care facilities continue to battle against the same causes year after year with little improvement. Experience and literature surrounding mechanical ventilation supports the concept that we struggle with unrecognized patient deterioration, alarm fatigue, opioidinduced respiratory depression, unplanned extubation or disconnects, and hospital-acquired conditions such as ventilator-associated pneumonia or ventilator-induced lung injury. ${ }^{51,52}$ Traditional monitoring assessments have shortcomings that negatively affect both patient outcomes and the efficiency of care. Our visual patient-ventilator assessment leaves our patients remotely monitored a large percent of the time (Fig. 4). This causes us to rely too heavily on alarms within the room or at a central station, and clinicians must monitor patients by sight and sound. Failure to capture clinically relevant ventilation changes or trends in measured parameters such minute ventilation, peak airway pressure, heart rate, pulse oximetry, and breathing frequency can threaten the safety culture we work so hard to create. Fortunately, key advancements in technology present significant opportunities to shift away from reactive and episodic monitoring in favor of comprehensive surveillance that may allow clinicians to intercept adverse events before they occur and well before an alarm.

Alarm Analytics. Since the introduction of biomedical device integration, several manufacturers have been developing systems that help address some of the alarm management issues of today. First, the concept that data are powerful is real. Prior to biomedical device integration, alarm policy compliance was manual and very labor-intensive. We know from our previous discussion that simple manual surveillance of alarm parameters can improve compliance, but what if we could do this in a less labor-intensive way? Today there are at least 3 manufacturers that offer the ability to monitor and track alarm compliance. Table 2 describes alarm management issues and potential solutions through advancements in human behavior and technology. 
Alarm Strategies and Surveillance for Mechanical Ventilation

Table 2. Alarm management issues with possible solutions

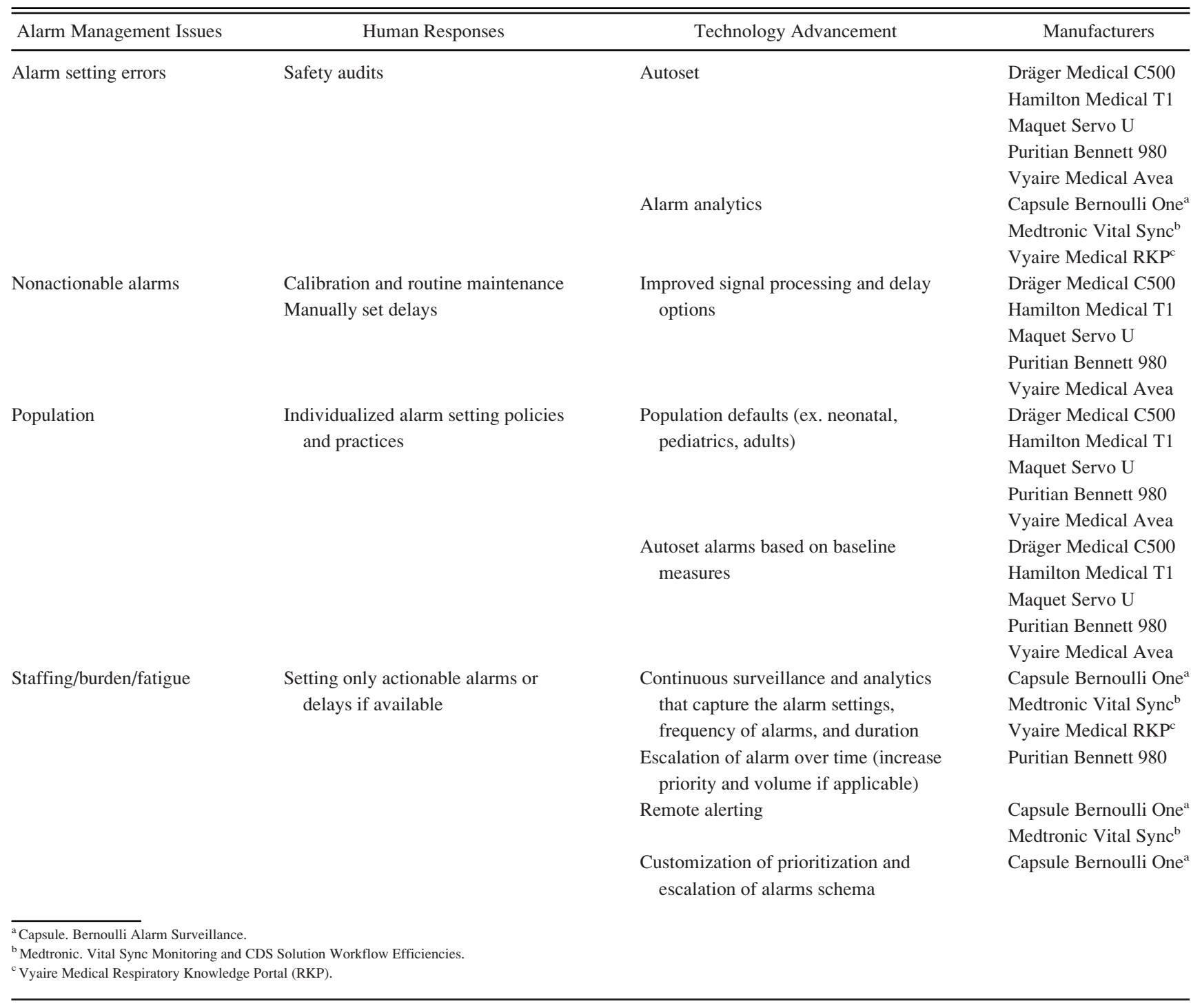

Smart Room. The concept of a smart room of event detection has been explored to improve the accuracy of alarms and to provide contextual assessments of monitoring readings. These smart hospital rooms of the future will be able to identify the presence of a health care provider, type of provider, family member, or patient movement. Additionally, these smart rooms theoretically would be able to identify vital missing components of today's hospital room, such as whether a staff member washed their hands, when someone entered and left the room, or the type and time a certain medication is being administered, and could intervene if an error were about to occur. In a study to enhance the safety of mechanically ventilated patients, Lins et $\mathrm{al}^{53}$ used an ambient process analysis to classify a patients' status, to detect a provider's presence and activity, and to monitor power supply. They were able to create a process to address real-life errors and block the clinician's ability to place the ventilator in stand-by mode while still connected to a patient. The authors' group was able to function with and without cameras, demonstrating the flexibility of the system to utilize differing technologies to address real concerns such as privacy.

Auditory Icons. In the ICU, where most patients receive mechanical ventilation, it is often difficult or impossible to audibly tell the difference between a heart rate alarm or a mechanical ventilator alarm, not to mention the multiple levels of alarms (eg, advisory to critical). This makes accurate and timely identification difficult, to say the least. Through an interprofessional collaboration of music engineering, medicine, and nursing, a research group at the University of Miami was able to come up with auditory icons to serve as metaphor for the event taking place. ${ }^{54}$ 
Table 3. Examples of auditory icon metaphors

\begin{tabular}{llll}
\hline \hline Alarm Category & \multicolumn{1}{c}{ Metaphor 1 } & \multicolumn{1}{c}{ Metaphor 2 } & Metaphor 3 \\
\hline Cardiovascular & Fast, rhythmic drum pattern & Several pulses of a lup-dup heart beat & Tick-tock of a clock \\
Ventilation & Sound of a science fiction ventilator mask & Inhalation followed by exhalation & HVAC system \\
Oxygenation & High-pressure build-up of air escaping a tank & 3 wine cork pops & Depressurization of a mask or tank \\
Equipment failure & Improper start of a cold motor (pull cord) & Improper start of a cold motor (pull & $\begin{array}{c}\text { Powering down of a science fiction } \\
\text { motor; synthesized }\end{array}$
\end{tabular}

Adapted from Reference 54.

HVAC $=$ heating, ventilation, and air conditioning system

Table 3 provides an example of alarms that are often encountered with mechanical ventilation. Through their work, they were able to identify the best-performing auditory icon alarms for each category and developed a set of 8 auditory icons to be proposed to relevant regulatory bodies and device manufacturers. If adoption is swift, care providers could identify an alarm or alert without seeing the ventilator or patient.

Alarm Prevention. The axiom "an ounce of prevention is worth a pound of cure" is just as true today as it was in Benjamin Franklin's time. It is obvious that mitigating the alarm is the best course of action, yet the "how" remains elusive. Manufacturers (eg, Capsule, Medtronic, and Vyaire) have created applications to help identify patient readiness to wean, ventilator protocol variations, alarm policy compliance, and ventilator-induced lung injury, to name a few. They have also incorporated other metrics of quality, such as ventilator-associated pneumonia and ventilator-associated events. We would all agree there is no better way to reduce alarms and therefore alarm fatigue than to prevent alarms by reducing adverse events or shortening the duration of mechanical ventilation.

Alarm Flooding. Alarm flooding is the phenomenon of presenting more alarms in a given period of time than a human operator can effectively respond to; this has been identified as the root cause of plant incidences such as the Texaco Pembroke refinery fire and the accident at the Three-Mile Island Nuclear Plant. ${ }^{55}$ Human performance has taught us that approximately one alarm per minute is the limit a typical person can handle, yet we know that alarms exceed this threshold frequently. ${ }^{56}$ Alarm flooding has not been effectively explored in health care, likely because of the inability to record all of the alarms efficiently until now; biomedical device integration may assist in additional research opportunities along these lines.

\section{Summary}

Current practices for alarm management and prevention can be improved by multiple available interventions and practices. These include targeted education with reinforcement, standardized alarm policies that respect patient differences and include peer follow-up, and the adoption of assistive technology. Benefits include improved patient safety, reduced clinician burden and fatigue, and enhanced efficiency which can lead to cost savings. Continued research is needed on human and environmental factors that influence alarm awareness and perception. Continually maximizing alarm prevention and utilization for mechanical ventilation as technology and care continue to advance in complexity are mandatory responsibilities.

\section{ACKNOWLEDGMENT}

The authors would like to thank Molly Quinn for her contribution, particularly to the origination challenges in alarm monitoring.

\section{REFERENCES}

1. Tume LN, Kneyber MC, Blackwood B, Rose L. Mechanical ventilation, weaning practices, and decision making in European PICUs. Pediatr Crit Care Med 2017;18(4):e182-e188.

2. Tobin M, Manthous C. Mechanical ventilation. Am J Respir Crit Care Med 2017;196(2):P3-P4.

3. Kacmarek RM, Pirrone M, Berra L. Assisted mechanical ventilation: the future is now. BMC Anesthesiol 2015;15(1):110.

4. 2019 top 10 health technology hazards executive brief: a report from health devices. Plymouth Meeting: ECRI Institute; 2018.

5. Kowalczyk L. Ventilator errors are linked to 119 deaths: warnings are often ignored, missed by overtaxed caregivers. Boston Globe. June 1, 2012.

6. Love LC, Millin CJ, Kerns CD. Take precautions with audible alarms on ventilators. Nursing 2011;41(9):65.

7. Ruppel H, Funk M, Whittemore R. Measurement of physiological monitor alarm accuracy and clinical relevance in intensive care units. Am J Crit Care 2018;27(1):11-21.

8. The Joint Commision. Hospital National Patient Safety Goals. Available at: https://www.jointcommission.org/en/standards/nationalpatient-safety-goals/hospital-2020-national-patient-safety-goals. Accessed April 1, 2020.

9. Hess DR, Kallstrom TJ, Mottram CD, Myers TR, Sorenson HM, Vines DL, et al. Care of the ventilator circuit and its relation to ventilator-associated pneumonia. Respir Care 2003;48(9):869-879.

10. MacIntyre NR, Cook DJ, Ely EW Jr, Epstein SK, Fink JB, Heffner JE, et al. Evidence-based guidelines for weaning and discontinuing ventilatory support: a collective task force facilitated by the American College of Chest Physicians; the American Association for Respiratory Care; 


\section{Alarm Strategies and Surveillance for Mechanical Ventilation}

and the American College of Critical Care Medicine. Chest 2001;120 (6):375S-395S.

11. American Association for Respiratory Care. AARC clinical practice guidelines: endotracheal suctioning of mechanically ventilated patients with artificial airways 2010. Respir Care 2010;55(6):758-764.

12. American Association for Respiratory Care. AARC clinical practice guidelines: long-term invasive mechanical ventilation in the home2007 revision \& update. Respir Care 2007;52(8):1056-1062.

13. McArthur CD. AARC clinical practice guidelines: capnography/capnometry during mechanical ventilation-2003 revision \& update. Respir Care 2003;48(5):534-539.

14. Chang DW. AARC clinical practice guidelines: in-hospital transport of the mechanically ventilated patient-2002 revision \& update. Respir Care 2002;47(6):721-723.

15. American Association for Respiratory Care. AARC clinical practice guideline. Patient-ventilator system checks. Respir Care 1992;37(8): 882-886.

16. University Health System Consortium, American Association for Respiratory Care. Safe initiation and management of mechanical ventilation white paper. Available at: https://www.aarc.org/aarc-partners-withuhc-on-mechanical-ventilation-white-paper. Accessed April 1, 2020.

17. Lawless ST. Crying wolf: false alarms in a pediatric intensive care unit. Crit Care Med 1994;22(6):981-985.

18. Chambrin MC, Ravaux P, Calvelo-Aros D, Jaborska A, Chopin C, Boniface B. Multicentric study of monitoring alarms in the adult intensive care unit (ICU): a descriptive analysis. Intensive Care Med 1999;25(12):1360-1366.

19. Tsien CL, Fackler JC. Poor prognosis for existing monitors in the intensive care unit. Crit Care Med 1997;25:614-619.

20. Siebig S, Kuhls S, Imhoff M, Gather U, Scholmerich J, Wrede CE. Intensive care unit alarms-how many do we need? Crit Care Med 2010;38(2):451-456

21. Cvach M. Monitor alarm fatigue: an integrative review. Biomed Instrum Technol 2012;46(4):268-277.

22. Bonafide CP, Lin R, Zander M, Graham CS, Paine CW, Rock W, et al. Association between exposure to nonactionable physiologic monitor alarms and response time in a children's hospital. J Hosp Med 2015;10(6):345-351

23. Pham JC, Williams TL, Sparnon EM, Cillie TK, Scharen HF, Marella WM. Ventilator-related adverse events: a taxonomy and findings from 3 incident reporting systems. Respir Care 2016;61(5):621-631.

24. Malkary G. Healthcare without bounds: trends in clinical surveillance and analytics. Menlo Park, CA: Spyglass Consulting Group; 2018.

25. Jungquist CR, Smith K, Nicely KL, Polomano RC. Monitoring hospitalized adult patients for opioid-induced sedation and respiratory depression. Am J Nurs 2017;117(3 Suppl 1):S27-S35.

26. Graham KC, Cvach M. Monitor alarm fatigue: standardizing use of physiological monitors and decreasing nuisance alarms. Am J Crit Care 2010;19(1):28-34.

27. Bennett $\mathrm{C}, \mathrm{McNeer} \mathrm{R}$, Leider C. Urgency analysis of audible alarms in the operating room. Presented at the 12th International Society for Music Information Retrieval Conference, held Oct 24-28, 2011, in Miami, Florida.

28. Bach TA, Berglund LM, Turk E. Managing alarm systems for quality and safety in the hospital setting. BMJ Open Qual 2018;7(3):e000202.

29. Lipton JA, Van Ettinger MJB, Barendse RJ, Van Dam TB, Van Der Putten NJHH, Nelwan SP. Alarms on the intensive cardiac care unit. Comput Cardiol 2009;36:253-256.

30. Joshi R, Mortel HV, Feijs L, Andriessen P, Pul CV. The heuristics of nurse responsiveness to critical patient monitor and ventilator alarms in a private room neonatal intensive care unit. PLoS One 2017;12(10): e0184567.

31. Cvach MM, Stokes JE, Manzoor SH, Brooks PO, Burger TS, Gottschalk A, Pustavoitau A. Ventilator alarms in intensive care units: frequency, duration, priority, and relationship to ventilator parameters. Anesth Analg 2018 [Epub ahead of print].

32. Dills CC. Managing mechanical ventilator alarms with middleware. Biomed Instrum Technol 2017;51(s2):62-65.

33. Drew BJ, Harris P, Zègre-Hemsey JK, Mammone T, Schindler D, Salas-Boni R, et al. Insights into the problem of alarm fatigue with physiologic monitor devices: a comprehensive observational study of consecutive intensive care unit patients. PLoS One 2014;9(10): e110274.

34. Xie H, Kang J, Mills GH. Clinical review: the impact of noise on patients' sleep and the effectiveness of noise reduction strategies in intensive care units. Crit Care 2009;13(2):208.

35. Bliss JP, Gilson RD, Deaton JE. Human probability matching behaviour in response to alarms of varying reliability. Ergonomics 1995;38 (11):2300-2312.

36. Edworthy J, Hellier E. Alarms and human behaviour: implications for medical alarms. Br J Anaesth 2006;97(1):12-17.

37. Ruppel H, Funk M, Clark JT, Gieras I, David Y, Bauld TJ, et al Attitudes and practices related to clinical alarms: a follow-up survey. Am J Crit Care 2018;27(2):114-123.

38. Alarm management becomes an NPSG. Hosp Peer Rev 2013;38 (9): $105-106$

39. Alarm management. Crit Care Nurse 2013;33(5):83-86

40. Management ED. ED accreditation update. Hospitals put on notice: alarm management is a top priority for 2014. ED Manag 2013;25(9):1-3.

41. Alarm management as a patient safety goal. Health Devices $2013 ; 42$ (8):242-247.

42. Alarm management gets renewed focus. Hosp Peer Rev 2013;38 (4):39-41.

43. Edworthy J. Alarms are still a problem. Anaesthesia 2013;68(8):791794.

44. Jacques S, Williams E. Reducing the safety hazards of monitor alert and alarm fatigue. Patient Safety Network. Available at: https://psnet. ahrq.gov/perspective/reducing-safety-hazards-monitor-alert-andalarm-fatigue. Accessed April 1, 2020.

45. Cvach M, Kitchens M, Smith K, Harris P, Flack MN. Customizing alarm limits based on specific needs of patients. Biomed Instrum Technol 2017;51(3):227-234.

46. Gorges M, Markewitz BA, Westenskow DR. Improving alarm performance in the medical intensive care unit using delays and clinical context. Anesth Analg 2009;108(5):1546-1552.

47. Ursprung R, Gray JE, Edwards WH, Horbar JD, Nickerson J, Plsek P, et al. Real time patient safety audits: improving safety every day. Qual Saf Health Care 2005; 14(4):284-289.

48. Sirgo Rodríguez G, Olona Cabases M, Martin Delgado MC, Esteban Reboll F, Pobo Peris A, Bodí Saera M, ART-SACC Study Experts. Audits in real time for safety in critical care: definition and pilot study. Med Intensiva 2014;38(8):473-482.

49. Christensen M. Noise levels in a general intensive care unit: a descriptive study. Nurs Crit Care 2007;12(4):188-197.

50. Makary MA, Daniel M. Medical error-the third leading cause of death in the US. BMJ 2016;353:i2139.

51. Morris TA, Gay PC, MacIntyre NR, Hess DR, Hanneman SK, Lamberti $\mathrm{JP}$, et al. Respiratory compromise as a new paradigm for the care of vulnerable hospitalized patients. Respir Care 2017;62(4):497-512.

52. Lee TW, Hong JW, Yoo JW, Ju S, Lee SH, Lee SJ, et al. Unplanned extubation in patients with mechanical ventilation: experience in the medical intensive care unit of a single tertiary hospital. Tuberc Respir Dis 2015;78(4):336-340.

53. Lins C, Gerka A, Lupkes C, Rohrig R, Hein A. Enhancing safety of artificially ventilated patients using ambient process analysis. Stud Health Technol Inform 2018;247:316-320.

54. Bennett C, Dudaryk R, Crenshaw N, Edworthy J, McNeer R. Recommendation of new medical alarms based on audibility, 


\section{Alarm Strategies and Surveillance for Mechanical Ventilation}

identifiability, and detectability in a randomized, simulation-based study. Crit Care Med 2019;47(8):1050-1057.

55. Niyazmand T, Izadi I. Pattern mining in alarm flood sequences using a modified PrefixSpan algorithm. ISA Trans 2019;90:287-293.
56. Reising DV, Downs JL, Bayn D. Human performance models for response to alarm notifications in the process industries: an industrial case study. Proceedings of the Human Factors and Ergonomics Society 48th Annual Meeting, Santa Monica, CA, 2004.

\section{Discussion}

Lamberti: It's been about 6 years since. The Joint Commission came out with that urgent plea. How do you think we're doing if you gave us a grade as an overall healthcare system about alarm fatigue. They said this is an urgent problem, let's fix it, and I'm just amazed that it's 6 years later and I don't think we're very far along.

Walsh: I would agree that we're not very far along. And I think we continue to do the same-old same-old but expecting different results. I'll give you an example. There are respiratory therapy departments that have refused to do monitoring of end-tidal $\mathrm{CO}_{2}$ on the floors for opioid depression or respiratory depression. Pharmacists have had to step up, and I've actually provided training to a pharmacy team to do end-tidal $\mathrm{CO}_{2}$ monitoring because nurses and respiratory therapy wouldn't pick it up. I thought what a shame. There's this disconnect because we're all about patient safety, but applause to my pharmacy colleagues who stepped up to the plate, learned the technology, so when they fulfill narcotics prescriptions they insured they were monitored appropriately. Again, I'm disappointed in my nursing and respiratory therapy colleagues, but that's how dire the straits in certain places because they don't value the safety net types of systems like end-tidal $\mathrm{CO}_{2}$. As Brady [Scott] went over, there are all other ways to monitor breathing frequency now and even remote alarming but people aren't picking up on it for some reason. I don't quite get it. This is a serious patient safety issue.

Scott: I agree with Brian, I think we're not doing a very good job with this. When we were writing the paper on mechanical ventilation alarms and alarm fatigue, ${ }^{1}$ the distressing part was that there wasn't a lot in the literature to write about. So, I think we have a long way to go. In terms of alarm fatigue, as you pointed out in your last slide, the \#1 attributing factor to alarm fatigue is frequency of the alarms. And I think a lot of the technology for secondary alarms is well intended, but the problem is that the secondary alarms on our cell phones or tablets are adding to the frequency of alarms. We simply don't know how to best set alarm thresholds yet. I think we need to seriously investigate the safest, most effective mechanical ventilator alarm thresholds, as the technology we have available is phenomenal. However, if we can't set it properly, it's just causing more and more alarms. We have a long way to go.

Lamberti: We need to understand that the alarm threshold that we chose determines the frequency of alarms. The low alarm threshold for oximetry of $90 \%$ was chosen by anesthesiologists 30 years ago in the OR, where patients have secure artificial airways. Dartmouth, who has published extensively on continuous pulse oximetry, sets their low threshold at $85 \%$ and sets a delay at $15 \mathrm{~s}$, getting rid of probably $80 \%$ of their alarms.

Scott: Another thing I wanted to comment on is the burden of alarms on patients. While we're hearing a lot of alarms as clinicians, so are patients. There have been some studies ${ }^{2,3}$ on how many times infants are subjected to these noises in the ICU and how it may be impacting development or how well they're sleeping. We should probably acknowledge that alarms are causing problems beyond clinicians and our issues with alarms fatigue.
Walsh: I did come across one study ${ }^{4}$ that looked at sleep in relationship to alarms, as well as sleep interruptions, and it was pretty eye-opening that they do contribute to interrupted sleep, particularly in the ICU.

Blanch: I think we are talking about digital medical devices, but the future is the digital transformation. The internet will change alarms at bedside. As an example, if a patient has a lifethreatening arrhythmia, a robot will carry stuff to the bedside before professionals have arrived. We need professionals with different disciplines to work with us in the ICU. We need humanized and technologically advanced ICUs. Medical devices are little computers and must be interoperable and interconnected. Experts said that the digital transformation of the health system will take 20 years from now to be completed. In the meantime, we must work to prevent motivation losing and professionals burnout.

Walsh: Thank you. In addition to what Brady was talking about and what you're talking about, we also need administrations to step up. And I mean that seriously because everyone wants to own the head of the bed and a lot of devices won't talk to another device. So, if you say, 'hey I'm not going to purchase you unless you talk to other devices or communicate with the EMR or remote alarm system', I bet manufacturers will get this straight if they want the sale. Often connectivity is an afterthought and then to get something to communicate with our remote alarm system or EHR sometimes takes months if not years to get connected. Then the products cycle out. To Brady's point about alarms, it's not just the patients but the families, they're being annoyed by these alarms as well and they notice when 
we don't respond to an alarm. It drives their stress level up because they don't have the knowledge to say, 'it's appropriate to ignore that one or it's not appropriate to ignore that one' so they can't determine a difference. So then you get irate families, bad customer service, those types of things also plaguing your hospital because you won't step up and control alarm management.

Blanch: I fully agree. This means we must change the way we approach the medical industry. Maybe, we should inquire to industry about solutions, not just buying medical devices, thus looking for better quality and efficiency.

Walsh: It may be going, by the way, like subscription-based software systems and those types of things where you subscribe for 6 months and then if you don't get the return to go with the competitor who's promising a similar return on investment. Versus buying all this hardware. I think the hardware will probably go away, we'll have standardized hardware hopefully, and it will be software-driven and we'll have products that make sense and help patients.

Smallwood: I have a question about the regulatory pathway for these solutions we're talking about. You spoke about some of the standards, for medical devices to be approved and sold in any country and the fact that they have to adhere what the government tells them to do and meet some standards. That's all well and good provided that everything I want to do is approved and is incorporated into the function of the device. But let's take a physiologic monitor for instance. If the monitor indeed has the capability for me, a random clinician, to go into the monitor and plug in my smart alarm that at my institution has been clinically accepted in this patient. What's the regulatory pathway to let me do this? Do I need approval? Do I need some holistic alarm solution or can I simply take some off the shelf parts, put them together the way I want and get the utility I'm looking for?

Walsh: One of the things we've done at the AARC is partner with AAMI on this. We were invited to join their multidisciplinary team to develop alarm awareness for mechanical ventilators. We've been working with that group. The group I presented who came up with different auditory icons, they worked through that group as well with ISO. If you don't know the regulatory pathway, AAMI makes recommendations to ISO. ISO is an international organization, AAMI is the American representative to that group so they take recommendations to try and get standards changed if they're not safe for our patients but they're limited obviously if the whole world doesn't agree we're kind of stuck. We can go to the FDA pathway and request different standards than ISO, and I know some manufacturers have done that. This innovative group, they worked within the ISO standards. So you'll still hear the beeps that are required by ISO, but then they added additional noises or auditory cues to actually tell you that this is a high priority alarm but it's also the heart rate alarm. They were working within the standards and ISO has actually accepted that and have said, 'yes, if you brought that to us today we would say it meets the standard' so I thought that was interesting. Of course they'll take that to the FDA when they go through their approval process. One of the things I thought was very innovative with this group is that they pointed out physiologic markers because they all had the same alarm. So pulse oximeter, heart rate, all had the same high priority beeps and they were actually able to differentiate between those different parameters they were monitoring that made it identifiable. I think it will promote patient safety tremendously if it gets passed and people start using it.

\section{REFERENCES}

1. Scott JB, De Vaux L, Dills C, Strickland SL. Mechanical ventilation and alarm fatigue. Respir Care 2019;64(10):1308-1313.

2. Belteki G, Morley CJ. Frequency, duration and cause of ventilator alarms on a neonatal intensive care unit. Arch Dis Child Fetal Neonatal Ed 2018;103(4):F307-F311.

3. Bergon-Sendin E, Perez-Grande C, LoraPablos D, De la Cruz Bertolo J, MoralPumarega MT, Bustos-Lozano G, PallasAlonso CR. Auditing of monitoring and respiratory support equipment in a level 3-c neonatal intensive care unit. Biomed Res Int 2015; 15:719497.

4. Salas RE, Gamaldo CE. Adverse effects of sleep deprivation in the ICU. Crit Care Clin 2008;24(3):461476, v-vi. 